\title{
Cubic surfaces and Borcherds products
}

\author{
Daniel Allcock* and Eberhard Freitag
}

\begin{abstract}
We apply Borcherds' methods for constructing automorphic forms to embed the moduli space $\mathcal{M}$ of marked complex cubic surfaces into $\mathbb{C} P^{9}$. Specifically, we construct 270 automorphic forms on the complex 4-ball $\mathcal{B}_{4}$, automorphic with respect to a particular discrete group $\Gamma$. We use the identification from [ACT2] of $\mathcal{M}$ with the Baily-Borel compactification of $\mathcal{B}_{4} / \Gamma$. Our forms span a 10-dimensional space, and we exhibit the image of $\mathcal{M}$ in $\mathbb{C} P^{9}$ as the intersection of 270 cubic hypersurfaces. Finally, we interpret the pairwise ratios of our forms as the original invariants of cubic surfaces, the cross-ratios introduced by Cayley. It turns out that this model of $\mathcal{M}$ was found by Coble [C] in an entirely different way; see [vG].
\end{abstract}

Mathematics Subject Classification (2000). 14J15, 11F55, $20 \mathrm{E} 40$.

Keywords. Moduli space, cubic surface, ball quotient, Borcherds product, automorphic form.

\section{Introduction}

The moduli space $\mathcal{M}$ of marked cubic surfaces can be identified with the BailyBorel compactification of $\mathcal{B}_{4} / \Gamma$, where $\mathcal{B}_{4}$ denotes the complex 4-ball and $\Gamma$ is a certain arithmetic reflection group. (See [ACT2] and also [ACT1].) In this paper we use the methods of $\mathrm{R}$. Borcherds to construct automorphic forms on $\mathcal{B}_{4}$. We will obtain an embedding of $\mathcal{M}$ into the 9-dimensional projective space $P^{9}(\mathbb{C})$, whose image is the intersection of 270 explicitly known cubic 8-folds. This map is compatible with the actions of the Weyl group $W\left(E_{6}\right)$ on $\mathcal{M}$ and $P^{9}$. The former action arises because $W\left(E_{6}\right)$ permutes the markings of cubic surfaces, and the latter action arises from the unique irreducible 10-dimensional representation of $W\left(E_{6}\right)$. Furthermore, the cubic 8-folds are all equivalent under $W\left(E_{6}\right)$.

The 10-dimensional linear system associated to this map into $P^{9}(\mathbb{C})$ contains 270 automorphic forms with known zeros, which play a central role in our investigation. In particular, there is a direct connection between them and the classical invariants of cubic surfaces introduced by Cayley. He considered the 27 lines on a smooth cubic surface and a certain configuration of 45 planes that they determine. By considering 4-tuples of these planes that meet along one of the 27 lines, Cayley

\footnotetext{
${ }^{*}$ Partially supported by NSF grant DMS 0070930.
} 
constructed 270 cross-ratios, and showed that these allow one to recover the original surface. We show that Cayley's cross-ratios coincide not with our Borcherds products but rather with the quotients of certain pairs of them. This relies on work of Naruki [Na] and is the main part of our proof that our map of $\mathcal{M}$ into $P^{9}$ is an embedding.

We are grateful to R. Borcherds, B. van Geemen, and R. Vakil for helpful discussions.

\section{The complex reflection group}

Let

$$
\mathcal{E}=\mathbb{Z}[\omega], \quad \omega=\sqrt[3]{1}=-\frac{1}{2}+\frac{\sqrt{-3}}{2},
$$

be the ring of Eisenstein integers. We consider the lattice

$$
\Lambda=\mathcal{E}^{1,4} \text {, }
$$

which is the $\mathcal{E}$-module $\mathcal{E}^{5}$ equipped with the hermitian form of signature $(1,4)$ given by

$$
\langle a, b\rangle=\bar{a}_{0} b_{0}-\bar{a}_{1} b_{1}-\cdots-\bar{a}_{4} b_{4} .
$$

Let $\operatorname{Aut}(\Lambda)$ be the unitary group of this lattice, i.e., the group of $\mathcal{E}$-module automorphisms which preserve the hermitian form. Complex conjugation acts as the identity on the residue field

$$
\mathbb{F}_{3}=\mathcal{E} / \sqrt{-3} \mathcal{E}
$$

which has order 3 , so the hermitian form induces a $\mathbb{F}_{3}$-valued quadratic form on the 5-dimensional $\mathbb{F}_{3}$-vector space $V=\Lambda / \sqrt{-3} \Lambda$. We denote the orthogonal group of $V$ by $\mathrm{O}(5,3)$ and define $\Gamma$ to be the kernel of the action of $\operatorname{Aut}(\Lambda)$ on $V$. We have the exact sequence

$$
1 \longrightarrow \Gamma \longrightarrow \operatorname{Aut}(\Lambda) \longrightarrow \mathrm{O}(5,3) \longrightarrow 1
$$

That is, $\Gamma$ is the congruence subgroup of $\operatorname{Aut}(\Lambda)$ of level $\sqrt{-3}$. For future reference we mention that $V$ contains 242 nonzero elements, of which 80 have norm 0,90 have norm 1 and 72 have norm -1 . Nonzero vectors in $V$ are equivalent under $\mathrm{O}(5,3)$ if and only if they have the same norm. The subgroup of $\mathrm{O}(5,3)$ generated by the reflections in the norm -1 vectors is isomorphic to the Weyl group $W\left(E_{6}\right)$, and $\mathrm{O}(5,3) \cong W\left(E_{6}\right) \times\{ \pm 1\}$. Furthermore, $W\left(E_{6}\right)$ contains a simple subgroup of index 2 and order 25920 .

A lattice vector $a \in \Lambda$ is called primitive if it cannot be divided in $\Lambda$ by a non-unit of $\mathcal{E}$. Also, $a$ is called

$$
\begin{array}{ll}
\text { isotropic (or null) } & \text { if }\langle a, a\rangle=0, \\
\text { a short root } & \text { if }\langle a, a\rangle=-1 \text {, or } \\
\text { a long root } & \text { if }\langle a, a\rangle=-2 .
\end{array}
$$


The roots are important because $\operatorname{Aut}(\Lambda)$ contains reflections in them. If $a$ is a short root and $\zeta$ is a unit of $\mathcal{E}$ (a sixth root of unity) then the map

$$
v \longmapsto v-(1-\zeta) \frac{\langle a, v\rangle}{\langle a, a\rangle} a
$$

is an automorphism of $\Lambda$. (In the special case $\zeta= \pm 1$ this is also true if $a$ is a long root.) This automorphism fixes the orthogonal complement of $a$ and maps $a$ to $\zeta a$. We call this automorphism a reflection if $\zeta \neq 1$. The order of a reflection is two, three or six, and we sometimes call reflections of these orders biflections, triflections and hexaflections. The third roots of unity are congruent to $1 \mathrm{mod}$ $\sqrt{-3}$, and therefore the triflections belong to the congruence group $\Gamma$. We remark that these triflections actually generate $\Gamma$ [ACT2], although we will not need this fact.

We need some information about the orbit structure of $\Lambda$ with respect to $\Gamma$. If $a, b \in \Lambda$ are in the same $\Gamma$-orbit, then their images in $V$ coincide. In some special cases the converse is true:

Proposition 2.1. Let $a$ and $b$ be two primitive isotropic vectors, or two short roots, or two long roots. Then $a$ and $b$ are equivalent under $\Gamma$ if and only if their images in $V$ coincide. The number of $\Gamma$-orbits of lines $\mathbb{C} a$, where a is a primitive isotropic vector, a short root or a long root, is 40, 36 or 45, respectively.

Proof. The "only if" part is trivial. To prove the converse, we use the fact that $\operatorname{Aut}(\Lambda)$ acts transitively on primitive isotropic vectors, on short roots, and on long roots (see Theorems 7.21 and 11.13 of [ACT2]). It is a general fact that if a group $G$ acts transitively on a set $X, N$ is a normal subgroup, and $x \in X$ has stabilizer $G_{x}$ in $G$, then the orbits of $N$ on $X$ are in 1-1 correspondence with the cosets in $G / N$ of the image therein of $G_{x}$. We apply this with $G=\operatorname{Aut}(\Lambda), N=\Gamma$, and $x$ a primitive isotropic vector, short root or long root of $\Lambda$. Then the number of $\Gamma$-orbits into which the $\operatorname{Aut}(\Lambda)$-orbit of $x$ splits is equal to the index in $\mathrm{O}(5,3)$ of the reduction modulo $\sqrt{-3}$ of $\operatorname{Aut}(\Lambda)_{x}$. We will now compute these reductions.

We first take $x$ to be a primitive null vector. According to paragraph 7.8 of $[\mathrm{ACT} 2]$, its stabilizer in $\operatorname{Aut}(\Lambda)$ contains as a normal subgroup a Heisenberg group with center $\operatorname{Im}(\mathcal{E})$ and central quotient $\mathcal{E}^{3}$, and the stabilizer modulo this Heisenberg group is the isometry group $(\mathbb{Z} / 6)^{3}: S_{3}$ of the lattice $\mathcal{E}^{3}$. (The notation ":" is ATLAS notation [Co] for a semidirect product.) By considering the matrices for these transformations, it is easy to see that the center of the Heisenberg group acts trivially on $V$, that $\mathcal{E}^{3}$ acts as $\mathcal{E}^{3} /\left(\sqrt{-3} \mathcal{E}^{3}\right) \cong(\mathbb{Z} / 3)^{3}$, that $(\mathbb{Z} / 6)^{3}$ acts as $(\mathbb{Z} / 2)^{3}$, and that $S_{3}$ acts faithfully. The image of the stabilizer in $\mathrm{O}(5,3)$ is a group $3^{3}: 2^{3}: S_{3}$, which has index 80 in $\mathrm{O}(5,3)$. Next we take $x$ to be a short root of $\Lambda$, say $(0,0,0,0,1)$, and $\bar{x}$ to be its image in $V$. Then the stabilizer of $\bar{x}$ is the orthogonal group of $\bar{x}^{\perp}$, which is generated by the reflections in the nonisotropic elements of $\bar{x}^{\perp}$. One can enumerate these vectors and check that each is the image of a root of $x^{\perp}$. The biflections in these roots reduce to reflections of $V$, proving 
that the stabilizer of $x$ in $\operatorname{Aut}(\Lambda)$ surjects to the stabilizer of $\bar{x}$, which has index 72 in $\mathrm{O}(5,3)$. Exactly the same argument applies if $x$ is a long root, say $(0,0,0,1,1)$, yielding an index of 90 .

We have shown that there are 80 (resp. 72, 90) orbits of primitive isotropic vectors (resp. short roots, long roots) in $\Lambda$, which is the same as the number of nonzero elements of $V$ of norm 0 (resp. -1, 1). Since the map from $\Gamma$-orbits of such lattice vectors to the corresponding set of vectors in $V$ is onto, it is bijective. This proves the first claim of the theorem, and the second follows immediately.

\section{The ball quotient}

The group $\Gamma$ acts on a complex 4 -ball in the projective space of $\mathbb{C}^{1,4}=\Lambda \otimes_{\mathcal{E}} \mathbb{C}$. We will describe this in some generality, for convenience in later sections. Let $\mathfrak{o}$ be an order in an imaginary quadratic number field. An o-lattice $L$ is a finitely generated projective o-module equipped with a Hermitian pairing $\langle$,$\rangle on L$ that takes value in the field of fractions of $\mathfrak{o}$. We take such pairings to be antilinear in the first and linear in the second variable. We say that $L$ is Lorentzian when its signature is $(1, n)$ with $n \geq 1$. A point of the projective space $P\left(L \otimes_{\mathfrak{o}} \mathbb{C}\right)$ is called positive if it is represented by a vector of positive norm. When $L$ is Lorentzian, the positive points form an open $n$-ball $\mathcal{B}(L)$ in projective space, which is also called the complex hyperbolic space of $L$. Aut $(L)$ acts properly discontinuously on $\mathcal{B}(L)$, and there is a natural compactification of the quotient, due to Baily and Borel [BB]. A cusp is an element of $P\left(L \otimes_{\mathfrak{o}} \mathbb{C}\right)$ that can be represented by an isotropic lattice vector. The cusps are the rational boundary points of $\mathcal{B}(L)$, and there are only finitely many orbits under $\operatorname{Aut}(L)$. We denote by $\mathcal{B}^{*}(L)$ the union of $\mathcal{B}(L)$ with the set of all cusps. The group $\operatorname{Aut}(L)$ acts on this extension, and the quotient of $\mathcal{B}^{*}(L)$ by any finite-index subgroup of $\operatorname{Aut}(L)$ carries the structure of a projective algebraic variety.

In our setting we have $\mathfrak{o}=\mathcal{E}$ and $L=\Lambda$. The hermitian form on $\mathbb{C}^{1,4}=\Lambda \otimes_{\mathcal{E}} \mathbb{C}$ is given by (2.1), and the identification of $\mathcal{B}(\Lambda)$ with the complex 4-ball is easy. Namely, any element of $\mathcal{B}(\Lambda)$ has a unique representative $z \in \mathbb{C}^{1,4}$ whose $z_{0^{-}}$ component is 1 . Considering the remaining coordinates identifies $\mathcal{B}(\Lambda)$ with the set of all $\left(z_{1}, \ldots, z_{4}\right) \in \mathbb{C}^{4}$ satisfying

$$
\left|z_{1}\right|^{2}+\cdots+\left|z_{4}\right|^{2}<1
$$

We will write $\mathcal{B}_{4}$ for $\mathcal{B}(\Lambda)$. We are interested in the quotient $X$ of $\mathcal{B}_{4}^{*}$ by $\Gamma \subset$ $\operatorname{Aut}(\Lambda)$. By Prop. 2.1, there are forty $\Gamma$-orbits of cusps in $\mathcal{B}_{4}^{*}$, so the boundary of the Baily-Borel compactification of $\mathcal{B}_{4} / \Gamma$ consists of 40 points.

If $a \in \Lambda$ has negative norm then the orthogonal complement $a^{\perp}$ of $a$ in $P\left(\mathbb{C}^{1,4}\right)$ meets $\mathcal{B}_{4}$ nontrivially, and we can also consider the intersection $a^{\perp} \cap \mathcal{B}_{4}^{*}$ and its image in $X$. It is known that the image is an algebraic subvariety of codimension one. We are particularly interested in this construction for $a$ a root of $\Lambda$; in this 
case we call $a^{\perp} \cap \mathcal{B}_{4}^{*}$ a mirror (of $\mathcal{B}_{4}^{*}$ ). The terminology derives from the fact that the mirror is the fixed-point set of the reflection(s) in $a$, and we call the mirror short or long according to whether $a$ is short or long. The image in $X$ of a short (long) mirror is called a short (long) mirror of $X$. For convenience we sometimes call a vector in $V$ a short (resp. long) vector if it has norm -1 (resp. 1). Note that $2=-1$ in $\mathbb{F}_{3}$. The short (long) vectors in $V$ are exactly the images of the short (long) roots of $\Lambda$. The short (long) mirrors in $X$ correspond to the 36 (45) pairs $\{ \pm a\}$ of short (long) vectors of $V$. We will need some results about the intersection behavior of mirrors. Orthogonality of mirrors in $\mathcal{B}_{4}^{*}$ is defined in the obvious way, and we call two mirrors in $X$ orthogonal if the corresponding vectors in $V$ are orthogonal. If two mirrors in $\mathcal{B}_{4}^{*}$ are orthogonal then so are their images in $X$. In fact we could take this as the definition of orthogonality of mirrors in $X$.

Lemma 3.1. Two short mirrors in $\mathcal{B}_{4}$ are either orthogonal or disjoint.

Proof. We take $x$ and $y$ to be short roots whose mirrors are the given mirrors. If the mirrors meet in $\mathcal{B}^{4}$ then a point of the intersection represents a positivedefinite one-dimensional subspace of $\mathbb{C}^{1,4}$. Its orthogonal complement is negative definite and contains $x$ and $y$. Hence the Gram matrix of $x, y$ must have positive determinant, so $\langle x, x\rangle\langle y, y\rangle-|\langle x, y\rangle|^{2}>0$, so $|\langle x, y\rangle|^{2}<1$. Since $\langle x, y\rangle \in \mathcal{E}$ we must have $\langle x, y\rangle=0$.

We now introduce the notion of a cross. This is fundamental for the paper because the automorphic forms we will construct vanish exactly along the points of a cross in $\mathcal{B}_{4}^{*}$. The word "cross" is meant to suggest mutual orthogonality.

Definition 3.2. $A$ cross in $V$ is a set of 5 pairwise orthogonal pairs $\pm a_{i}$, one pair consisting of long vectors and the others consisting of short vectors. The associated cross in $X$ is the union of the mirrors of the $\pm a_{i}$; it follows that a cross in $X$ is a set of 5 pairwise orthogonal mirrors, one long and 4 short. The associated cross in $\mathcal{B}_{4}^{*}$ is the preimage of the cross in $X$. A point of $\mathcal{B}_{4}^{*}$ lies in this cross just if it is orthogonal to a root whose projection to $V$ is one of the $\pm a_{i}$.

Since the three types of cross are in natural bijection, we will pass between them without comment.

Lemma 3.3. There are 135 crosses, three containing each of the 45 long mirrors of $X$, and the 135 crosses are all equivalent under $\mathrm{O}(5,3)$. More precisely, if $\ell$ is a long mirror in $X$ then the 12 short mirrors orthogonal to $\ell$ decompose in a unique way into three sets of 4 mirrors which are pairwise orthogonal, and the stabilizer of $\ell$ in $\mathrm{O}(5,3)$ permutes these sets transitively.

Proof. The transitivity of $\mathrm{O}(5,3)$ on crosses in $V$ is obvious, and the rest is just a 
calculation. By symmetry it suffices to check this for a single long vector $a$, say $(1,0,0,0,0)$. Then the three classes are

$$
\begin{aligned}
& \{ \pm(0,1,0,0,0), \pm(0,0,1,0,0), \quad \pm(0,0,0,1,0), \quad \pm(0,0,0,0,1)\}, \\
& \{ \pm(0,-1,1,1,1), \pm(0,1,-1,1,1), \pm(0,1,1,-1,1), \quad \pm(0,1,1,1,-1)\}, \\
& \{ \pm(0,1,1,1,1), \quad \pm(0,1,1,-1,-1), \pm(0,1,-1,1,-1), \pm(0,1,-1,-1,1)\} .
\end{aligned}
$$

The purpose of the following theorem is to allow us to prove in section 4 that the automorphic forms we construct there have no common zeros.

Theorem 3.4. No point of $X$ lies on all 135 crosses. Suppose $p$ is the point of $\mathcal{B}_{4}$ represented by $(1,0,0,0,0) \in \Lambda$ and $\bar{p}$ is its image in $X$. Then $\bar{p}$ is the only point of $X$ that lies on all the crosses containing $\bar{p}$. Finally, for each boundary point $\bar{b}$ of $X, \bar{b}$ is the only point of $X$ that lies on all the crosses containing $\bar{b}$.

In order to prove the theorem we will need to understand the $\Gamma$-orbits of points of $\mathcal{B}_{4}$ that, like $p$, lie on four short mirrors. If $q$ is such a point, then $q^{\perp}$ is a copy of the unimodular lattice $\mathcal{E}^{0,4}$, and it follows that $q$ is represented by a lattice vector of norm 1, and indeed by six such vectors. The images in $V$ of these vectors and of the short roots of $q^{\perp}$ form a cross, which we call the cross associated to $q$.

Lemma 3.5. The map just defined, which associates a cross to each point of $\mathcal{B}_{4}$ that lies on 4 short mirrors, defines a bijection from the set of $\Gamma$-orbits of such points to the set of crosses. If each of $p, q \in \mathcal{B}_{4}$ lies on four short mirrors, and the images in $V$ of the short roots of $p^{\perp}$ coincide with the images of the short roots of $q^{\perp}$, then $p$ and $q$ are $\Gamma$-equivalent.

Proof. For the first claim one uses the argument of Prop. 2.1. The essential facts are that $\operatorname{Aut}(\Lambda)$ acts transitively on such points of $\mathcal{B}_{4}$ and that the stabilizer in Aut $(\Lambda)$ of such a point of $\mathcal{B}_{4}$ is $(\mathbb{Z} / 6) \times(\mathbb{Z} / 6)^{4}: S_{4}$, which reduces modulo $\sqrt{-3}$ to $(\mathbb{Z} / 2)^{5}: S_{4}$, of index 135 in $\mathrm{O}(5,3)$. (The transitivity statement follows from the fact that such points in $\mathcal{B}_{4}$ correspond bijectively to the decompositions of $\Lambda$ as a direct sum $\mathcal{E}^{1,0} \oplus \mathcal{E}^{0,4}$.) The second claim is a consequence of the first: the short vectors of a cross determine the cross uniquely, so the crosses associated to $p$ and $q$ coincide.

Proof of Theorem 3.4. Most of the proof consists of computer calculations concerning combinatorics in $V$; we will describe the ideas in sufficient detail for them to be reproduced easily.

One can enumerate the roots orthogonal to $p$, and their images in $V$. A cross contains $p$ just if it contains one of these images. One can compute the set $\mathcal{C}$ of crosses satisfying this condition, and one finds $|\mathcal{C}|=69<135$. In particular, $p$ does not lie on all 135 crosses. Now we will show that $p$ is the only point of $\mathcal{B}_{4}$ (up 
to $\Gamma$-equivalence) that lies on all the crosses containing $p$. Suppose $q \in \mathcal{B}_{4}$ lies on every cross of $\mathcal{C}$; we will show that $q$ is $\Gamma$-equivalent to $p$. First we will show that $q$ lies on 4 short mirrors. Otherwise, the short roots orthogonal to $q$ project into some triple $T$ of mutually orthogonal antipodal pairs of short vectors of $V$. If $q$ lies on every cross in $\mathcal{C}$ then there is a way to choose a root in $q^{\perp}$ for each $C \in \mathcal{C}$, such that the image in $V$ of the root is one of the vectors of $C$. In particular, there is a way to choose an element $v \in C$ for each $C \in \mathcal{C}$, such that (1) if $v$ is short then $v \in T$, and (2) the span of all the $v$ 's has dimension at most 4. For each of the 540 possibilities for $T$ one can count the number of ways to choose vectors $v$ satisfying (1) and (2). It turns out that there are no ways to make such a choice, and it follows that $q$ cannot lie on only 3 (or fewer) short mirrors.

We have shown that the short roots of $q^{\perp}$ project onto some quadruple of mutually orthogonal antipodal pairs of short vectors of $V$, which we will denote by $T$. As in the previous paragraph, there is a way to choose an element $v \in C$ for each $C \in \mathcal{C}$, such that (1) and (2) are satisfied. For each of the 135 possibilities for $T$, one can count the number of ways to make such a set of choices. It turns out that for only one quadruple is there a way to do this, and this quadruple consists of $\pm(0,1,0,0,0), \ldots, \pm(0,0,0,0,1)$. Therefore the images in $V$ of the short roots of $q^{\perp}$ are these 8 vectors. Since these are also the images of the short roots of $p^{\perp}$, the $\Gamma$-equivalence of $p$ and $q$ follows from Lemma 3.5.

Now we turn to the boundary points of $X$. If $b$ is a boundary point of $\mathcal{B}_{4}^{*}$ then we may represent it by a primitive isotropic lattice vector $w$, and a cross contains $b$ just if it contains the image in $V$ of a root orthogonal to $w$. One can check that every nonisotropic element of $V$ that is orthogonal to the image $\bar{w}$ of $w$ in $V$ is the image of a root in $w^{\perp}$. (This is easy to check for any given $w$, and the result follows for general $w$ because of the transitivity of Aut $\Lambda$.) It follows that the set of crosses $\mathcal{C}_{b}$ containing $b$ consists of the crosses which contain a vector of $V$ orthogonal to $\bar{w}$. It is easy to compute the sets $\mathcal{C}_{b}$ for each of the 40 orbits of boundary points, and to check that no $\mathcal{C}_{b}$ is a subset of $\mathcal{C}$. This proves the second claim of the theorem. The first claim then follows, because no point of $X$ except for the image of $p$ lies on every cross in $\mathcal{C}$, and this point lies on only 69 of the 135 crosses.

Now we show that no point of $\mathcal{B}_{4}$ lies on all the crosses in $\mathcal{C}_{b}$, for any boundary point $b$. By symmetry it suffices to treat just one $\mathcal{C}_{b}$, and the proof is almost identical to the one used above. If $q \in \mathcal{B}_{4}$, then the short roots of $q^{\perp}$ project into some quadruple $T$ of mutually orthogonal short vectors of $V$. If $q$ lies on every cross in $\mathcal{C}_{b}$ then there is a way to choose an element $v \in C$ for each $C \in \mathcal{C}_{b}$, such that (1) and (2) are satisfied. An enumeration shows that there is no way to make such a choice, and the claim follows. Finally, it is easy to compare the $\mathcal{C}_{b}$ 's with each other as $b$ varies over the ( $\Gamma$-orbits of) boundary points, and check that none of the $\mathcal{C}_{b}$ 's contains any other. It follows that for each boundary point $\bar{b}$ of $X, \bar{b}$ is the only point of $X$ that lies on all the crosses containing $\bar{b}$. This completes the proof. 
We verified the enumerations with a computer program written in $\mathrm{C}++$, which ran to completion in less than a minute. Repeatedly checking condition (2) required more than $4 \times 10^{8}$ row-reduction operations, and we did this efficiently by enumerating the $3^{5}$ elements of $V$ and preparing a lookup table of all $3^{5 \cdot 2}$ possible row-reductions.

\section{Automorphic forms on the ball}

Borcherds has given two constructions for automorphic forms on $\mathrm{O}(2, n)$, which we will use to build automorphic forms on the 4-ball. Here we will use his additive lift [Bo1, §14], which generalizes correspondences of Shimura, Doi-Naganuma, Maass, Gritsenko, and others. In the next section we will discuss his other construction, which uses infinite products.

We begin in the setting of section 3 , with $\mathfrak{o}$ an order in an imaginary quadratic number field, $L$ an $\mathfrak{o}$-lattice of signature $(1, n), \mathcal{B}(L)$ the associated ball in projective space, and $\mathcal{B}^{*}(L)$ the union of the ball with the cusps. We assume that $L$ is integral (all inner products lie in $\mathfrak{o}$ ) and that $n>1$, so that $L$ has dimension at least 3. We define $\tilde{\mathcal{B}}(L)$ and $\tilde{\mathcal{B}}^{*}(L)$ to be the preimages of $\mathcal{B}(L)$ and $\mathcal{B}^{*}(L)$ in $L \otimes_{\mathfrak{o}} \mathbb{C}$. If $G$ is a subgroup of $\operatorname{Aut}(L)$ and $v: G \rightarrow S^{1} \subset \mathbb{C}^{\bullet}=\mathbb{C}-\{0\}$ is a character of $G$ then an automorphic form of weight $k \in \mathbb{Z}$ with respect to $G$ and $v$ is a holomorphic function $f: \tilde{\mathcal{B}}(L) \rightarrow \mathbb{C}$ satisfying

$$
f(t z)=t^{-k} f(z) \text { for } t \in \mathbb{C}^{\bullet}
$$

and

$$
f(\gamma z)=v(\gamma) f(z) \text { for } \gamma \in G .
$$

(If $n$ were 1 , so that $\mathcal{B}(L)$ were one-dimensional, then we would impose an additional condition of regularity at the cusps.) We denote the space of all such forms by $[G, k, v]$, or by $[G, k]$ if $v$ is trivial.

One can extend an automorphic form $f: \tilde{\mathcal{B}}(L) \rightarrow \mathbb{C}$ to $\tilde{\mathcal{B}}^{*}(L)$ in a natural way, providing boundary values for $f$. If $a$ is an isotropic element of $\tilde{\mathcal{B}}^{*}(L)$, so that it represents a cusp, then by the non-degeneracy of $\langle\cdot, \cdot\rangle$ we may choose $b \in L \otimes_{\mathfrak{o}} \mathbb{C}$ satisfying $\langle b, a\rangle \neq 0$. For all $\tau \in \mathbb{C}$ with sufficiently large imaginary part, $\tau a+2 \mathrm{i}\langle b, a\rangle b$ has positive norm. The limit

$$
f(a):=\lim _{\Im \tau \rightarrow \infty} f(\tau a+2 \mathrm{i}\langle b, a\rangle b)
$$

exists and is independent of the choice of $b$. This follows from the Fourier Jacobi expansion of $f$ at a cusp; we refer to [Sh] for more details.

An automorphic form $f \in[G, k, v]$ is of course not a function on $\mathcal{B}(L)$ unless $k=0$. But it is clear that the zero-locus of $f$ is preserved by $G$ and scalar multiplication, so the set of zeros of $f$ in $\mathcal{B}^{*}(L) / G$ is well-defined. It is a closed algebraic subvariety of pure codimension one. 
Now we will discuss Borcherds' additive lift. We consider the $\mathbb{Z}$-lattice $M$ underlying $L$, which is the underlying $\mathbb{Z}$-module equipped with the even integral bilinear form

$$
(a, b):=\langle a, b\rangle+\langle b, a\rangle,
$$

which has signature $(2,2 n)$. The dual lattice with respect to $(\cdot, \cdot)$ is denoted $M^{\prime}$, and $M^{\prime} / M$ is a finite group. We remark that if $\alpha, \beta \in M^{\prime} / M$ then $(\alpha, \alpha)$ and $(\beta, \beta)$ are well-defined modulo 2 , while $(\alpha, \beta)$ is well-defined modulo 1 . The group $\mathrm{SL}(2, \mathbb{Z})$ acts on the group ring $\mathbb{C}\left[M^{\prime} / M\right]$ by means of the Weil representation $\varrho_{M}$, which is defined in terms of the standard generators

$$
T=\left(\begin{array}{ll}
1 & 1 \\
0 & 1
\end{array}\right) \quad \text { and } \quad S=\left(\begin{array}{cc}
0 & -1 \\
1 & 0
\end{array}\right)
$$

by

$$
\begin{aligned}
& \varrho_{M}(T) e_{\alpha}=\exp (\pi \mathrm{i}(\alpha, \alpha)) e_{\alpha}, \\
& \varrho_{M}(S) e_{\alpha}=\frac{\mathrm{i}^{n-1}}{\sqrt{\left|M^{\prime} / M\right|}} \sum_{\beta \in M^{\prime} / M} \exp (-2 \pi \mathrm{i}(\alpha, \beta)) e_{\beta} .
\end{aligned}
$$

(We denote the standard generators of the group $\operatorname{ring} \mathbb{C}\left[M^{\prime} / M\right]$ by $e_{\alpha}$, with $\alpha$ varying over $M^{\prime} / M$.) The Weil representation factors through $\operatorname{SL}(2, \mathbb{Z} / N \mathbb{Z})$, where $N$ is the smallest natural number such that $\frac{N}{2}(a, a)$ is integral for all $a \in M^{\prime}$.

The inputs of Borcherds' additive lift are vector valued modular forms $f: H \rightarrow$ $\mathbb{C}\left[M^{\prime} / M\right]$ on the usual upper half plane $H$ with respect to the Weil representation. More precisely, we require that $f=\left(f_{\alpha}\right)_{\alpha \in M^{\prime} / M}$ satisfy

$$
\begin{gathered}
f_{\alpha}(\tau+1)=e^{\pi \mathrm{i}(\alpha, \alpha)} f_{\alpha}(\tau), \\
f_{\alpha}\left(-\frac{1}{\tau}\right)=\tau^{k+1-n} \frac{\mathrm{i}^{n-1}}{\sqrt{\left|M^{\prime} / M\right|}} \sum_{\beta \in M^{\prime} / M} e^{-2 \pi \mathrm{i}(\alpha, \beta)} f_{\beta}(\tau), \text { and }
\end{gathered}
$$

$f$ is holomorphic at the cusp infinity.

The weight of $f$ is the exponent $k+1-n$ of $\tau$ in the second condition. While Borcherds' additive lift allows inputs which have poles at the cusps, we do not need this extension. But even in the case of modular forms which are regular at the cusps, Borcherds extended previous constructions because he imposes no restriction on the weight of $f$, and does not require that $f$ be a cusp form.

The additive lift is a linear map $\Psi$ from the space of such $f$ into a certain space of automorphic forms on $\mathcal{B}(L)$. We give its important properties in the following theorem, which is a specialization of Theorem 14.3 in $[\mathrm{Bo} 1]$ to $\mathrm{U}(1, n) \subseteq \mathrm{O}(2,2 n)$.

Theorem 4.1. Let $G$ be the subgroup of $\operatorname{Aut}(L)$ that acts trivially on $M^{\prime} / M$. There exists a linear map $\Psi$ (the additive lift) from the space of elliptic modular forms with the properties 1-3 above into the space $[G, k]$ of automorphic forms of weight $k$ with respect to $G$ and the trivial character. This lifting is equivariant 
with respect to the action of $\operatorname{Aut}(L)$. (Aut $(L)$ acts on $[G, k]$ because $G$ is normal in $\operatorname{Aut}(L)$, and on the space of elliptic modular forms via its action on $M^{\prime} / M$.)

Furthermore, Borcherds shows how to compute the values of $\Psi(f)$ at the cusps of $\tilde{\mathcal{B}}^{*}(L)$ from the Fourier coefficients of $f$.

We now turn to the case of interest, with $\mathfrak{o}=\mathcal{E}$ and $L=\Lambda$. The $\mathbb{Z}$-lattice underlying the 1-dimensional lattice $\mathcal{E}$ is the $A_{2}$ root lattice (the hexagonal lattice in the plane with minimal norm 2), which has index 3 in its dual. From the definition of $\Lambda$ as a direct sum, we see that $M^{\prime} / M$ has order $3^{5}$. Indeed more is true: $M^{\prime}$ coincides with $(\sqrt{-3})^{-1} \Lambda$, so that $M^{\prime} / M$ is canonically isomorphic to the $\mathbb{F}_{3}$-vector space $V=\Lambda / \sqrt{-3} \Lambda$ introduced in section 2 . In particular, $G$ is the congruence subgroup $\Gamma$. One can check that if $\alpha, \beta \in M^{\prime} / M$ then $(\alpha, \beta)$ is $0,2 / 3$ or $-2 / 3$ (modulo 1 ) according to whether the corresponding elements of $V$ have inner product 0,1 or -1 (in $\mathbb{F}_{3}$ ). Similarly, if $\alpha \in M^{\prime} / M$ then $(\alpha, \alpha)$ is $0,2 / 3$ or $-2 / 3$ (modulo 2) according to whether the corresponding element of $V$ has norm 0,1 or -1 . It follows from this that the level of the Weil representation is $N=3$, so that the representation factors through $\mathrm{SL}\left(2, \mathbb{F}_{3}\right)$. We will usually write $V$ in place of $M^{\prime} / M$ to lighten the notation.

We apply Theorem 4.1 in the simplest case, where $f$ is a modular form of weight 0 , hence a constant, which is to say an element of $\mathbb{C}[V]^{\mathrm{SL}\left(2, \mathbb{F}_{3}\right)}$. The weight being 0 means that $1-k+n=0$, so that $k=n-1=3$. Therefore Borcherds' additive lift gives a linear map

$$
\mathbb{C}[V]^{\mathrm{SL}\left(2, \mathbb{F}_{3}\right)} \longrightarrow[\Gamma, 3] .
$$

We remark that since $\Gamma$ contains the cube roots of unity acting as scalars, every automorphic form on $\tilde{\mathcal{B}}_{4}$ for $\Gamma$, with trivial character has weight divisible by 3 . Our first task is to find some elements of $\mathbb{C}[V]^{\mathrm{SL}\left(2, \mathbb{F}_{3}\right)}$.

Lemma 4.2. Let $a_{0}, \ldots, a_{4}$ be an orthogonal basis for $V$ consisting of one long vector and four short vectors, and let $C=\left(C_{\alpha}\right)_{\alpha \in V} \in \mathbb{C}[V]$ be defined by the condition that $C_{\alpha}$ is the complex number 1,0 or -1 according to whether $\prod_{i}\left(\alpha, a_{i}\right)$ is the element 1,0 or -1 of $\mathbb{F}_{3}$. Then $C$ is invariant under the Weil representation. Furthermore, $C$ changes sign under reflection in any of the $a_{i}$, and is characterized in $\mathbb{C}[V]$, up to a scalar, by this property.

To avoid the impression that the $C$ 's were discovered by clever guesswork, we should mention that we found this construction quite late, following extensive computer work.

Proof. The behavior of $C$ under the reflections is obvious, and the invariance under $\mathrm{SL}\left(2, \mathbb{F}_{3}\right)$ may be checked by a computer calculation. To see the last claim, suppose $D=\left(D_{\alpha}\right) \in \mathbb{C}[V]$ has the stated property. If $\alpha$ is orthogonal to one of the $a_{i}$ then we have $D_{\alpha}=-D_{\alpha}$ by the transformation rule, so that $D_{\alpha}=0$. The remaining $\alpha$ fall into a single orbit of size 32 under the group $(\mathbb{Z} / 2)^{5}$ generated by the reflections 
in the $a_{i}$, so all the remaining $D_{\alpha}$ are determined by any one of them.

It is easy to work this out explicitly in an example: if $a_{0}, \ldots, a_{4}$ are $(1,0, \ldots, 0)$, $\ldots,(0, \ldots, 0,1)$ then $C$ is supported on those $\alpha$ of the form $( \pm 1, \ldots, \pm 1)$, with $C_{\alpha}=+1$ or -1 according to whether there are an even or odd number of minus signs. Note that $C$ is supported on the isotropic vectors in $V$, which is not immediately obvious from the construction. It follows from the lemma and Theorem 4.1 that to each cross there is associated an automorphic form on $\mathcal{B}_{4}$, well-defined up to sign. We will see below that the zero-locus of this form is exactly the associated cross in $\mathcal{B}_{4}$. To resolve the sign ambiguity it is convenient to introduce the notion of a signed cross. This is just a basis $\left\{a_{0}, \ldots, a_{4}\right\}$ as in the lemma, modulo the equivalence relation that $\left\{a_{0}, \ldots, a_{4}\right\} \sim\left\{a_{0}^{\prime}, \ldots, a_{4}^{\prime}\right\}$ if the $a_{i}^{\prime}$ differ from the $a_{i}$ by a permutation and evenly many sign changes. It is clear that there are two signed crosses for every cross, and that the lemma assigns an element of $\mathbb{C}[V]$ to each of the 270 signed crosses.

Lemma 4.3. The space

$$
\mathbb{C}\left[M^{\prime} / M\right]^{\mathrm{SL}(2, \mathbb{Z})}=\mathbb{C}[V]^{\mathrm{SL}\left(2, \mathbb{F}_{3}\right)}
$$

has dimension 10 and is spanned by the elements of $\mathbb{C}[V]$ associated to the signed crosses. The group $\mathrm{O}(5,3)$ acts irreducibly on this space, with $W\left(E_{6}\right)$ acting by its unique 10-dimensional irreducible representation and the central involution acting by -1 . The multiplicity of this representation in $\mathbb{C}[V]$ is one.

Proof. It is easy to make a computer construct the elements $C$ of $\mathbb{C}[V]$ associated to the signed crosses and check that their complex span $Z$ is 10-dimensional. Consulting the character table shows that any 10-dimensional representation of $W\left(E_{6}\right)$ is either trivial, or the irreducible representation in 10 dimensions, or else the sum of the (unique) irreducible 6-dimensional representation and a 4dimensional trivial one. These may be distinguished by the trace of almost any group element, say a short reflection $R$, which has ATLAS [Co] conjugacy class 2C. The fixed space of $R$ in $Z$ is spanned by the vectors $C+R(C)$ where $C$ is as above. It is easy to check that this space has dimension 5 , so that $R$ has trace 0 , so that $W\left(E_{6}\right)$ acts irreducibly on $Z$. It is obvious that each $C$ changes sign under the central involution of $\mathrm{O}(5,3)$. If the multiplicity of this $\mathrm{O}(5,3)$-representation in $\mathbb{C}[V]$ were more than one, then the subspace of $\mathbb{C}[V]$ that changed sign under the reflections of each vector in a cross would have dimension $>1$, contrary to Lemma 4.2 .

To see that $Z$ is all of $\mathbb{C}[V]^{\mathrm{SL}\left(2, \mathbb{F}_{3}\right)}$, suppose $C=\left(C_{a}\right)_{a \in V}$ is an element of $\mathbb{C}[V]^{\mathrm{SL}\left(2, \mathbb{F}_{3}\right)}$. Invariance under $T$ means that $C$ is supported on the 81 isotropic elements. Invariance under $S^{2}=-I$ means $C_{-a}=-C_{a}$. Invariance under $S$ can be read as a linear equation in 40 indeterminates, and it is easy to make a computer check that the space of solutions has only 10 dimensions. If one is prepared to do more work with group characters, one can of course find the 
complete decomposition of $\mathbb{C}[V]$ under $\operatorname{SL}\left(2, \mathbb{F}_{3}\right) \times \mathrm{O}(5,3)$; this is done in $[\mathrm{Fr}]$.

We will write $W$ for the image of Borcherds' additive lift $\mathbb{C}[V]^{\mathrm{SL}\left(2, \mathbb{F}_{3}\right)} \rightarrow[\Gamma, 3]$. Our next theorem asserts that the automorphic forms we have constructed are nontrivial:

Proposition 4.4. Borcherds' additive lift

$$
\mathbb{C}[V]^{\mathrm{SL}\left(2, \mathbb{F}_{3}\right)} \rightarrow W \subset[\Gamma, 3]
$$

is an $\mathrm{O}(5,3)$-equivariant embedding.

Proof. The $\mathrm{O}(5,3)$-equivariance is part of Theorem 4.1. To prove injectivity, we construct an inverse by using the boundary values of the automorphic forms. Namely, if $f \in[\Gamma, 3]$ then we define $C=\left(C_{\alpha}\right)_{\alpha \in V}$ by taking $C_{\alpha}=0$ if $\alpha$ is zero or nonisotropic, and $C_{\alpha}=f(\tilde{\alpha})$ otherwise, where $\tilde{\alpha}$ is any primitive isotropic vector in $\Lambda$ representing $\alpha$. This definition is independent of the choice of $\tilde{\alpha}$ because $f$ is $\Gamma$-invariant and all the primitive isotropic preimages of $\alpha$ are $\Gamma$-equivalent (Prop. 2.1). The irreducibility of $\mathbb{C}[V]^{\mathrm{SL}\left(2, \mathbb{F}_{3}\right)}$ as an $\mathrm{O}(5,3)$-module and the fact that its multiplicity in $\mathbb{C}[V]$ is one imply that the composition

$$
\mathbb{C}[V]^{\operatorname{SL}\left(2, \mathbb{F}_{3}\right)} \stackrel{\text { additive lift }}{\longrightarrow} W \subset[\Gamma, 3] \stackrel{\text { boundary values }}{\longrightarrow} \mathbb{C}[V]
$$

is a scalar. The problem is to show that this scalar is nonzero. This is a straightforward but tedious calculation using Borcherds' formulae for the Fourier expansions of additive lifts ([Bo1], 14.3) and the explicit embedding of $\mathcal{B}_{4}$ into the hermitian symmetric space of $\mathrm{O}(2,8)$. The latter space consists of two-dimensional positive definite real subspaces of $M \otimes_{\mathbb{Z}} \mathbb{R}$. Every positive definite complex line in $\Lambda \otimes_{\mathcal{E}} \mathbb{C}$ (i.e. a point in $\mathcal{B}_{4}$ ) defines such a subspace. One has to express this embedding in the coordinates which Borcherds uses in his theorem 14.3. Details of this calculation can be found in section 6 of $[\mathrm{Fr}]$.

Lemma 4.3 shows that our 270 automorphic forms satisfy many linear equations. Some of these are easy to see, and those treated in the following lemma will receive a geometric interpretation in section 7 . To formulate the lemma we note that there is an $\mathrm{O}(5,3)$-invariant inner product on $W$, which is unique up to scale by the irreducibility of the representation.

Lemma 4.5. Let $v$ be a long vector of $V$. Then the automorphic forms associated to the six signed crosses involving $v$ lie in a 2-dimensional subspace of $W$, and form a scaled copy of the $A_{2}$ root system, i.e., the vertices of a regular hexagon centered at 0.

Proof. One can check this by computing the inner products of the 6 elements of 
$\mathbb{C}[V]$, using the restriction of the inner product

$$
\left(\left(C_{\alpha}\right),\left(D_{\alpha}\right)\right)=\sum_{\alpha \in V} C_{\alpha} D_{\alpha}
$$

which is obviously $\mathrm{O}(5,3)$-invariant and therefore the natural inner product. But here is a conceptual argument. The reflection $R$ in $v$ is not in the simple subgroup of $W\left(E_{6}\right)$, but $-R$ is, and has conjugacy class $2 A$ in ATLAS notation. Consulting the character table shows that $-R$ has trace -6 , so that the subspace $Z$ of $W$ that $R$ negates has dimension 2. Lemma 4.2 associates to each of the three crosses a one-dimensional subspace of $Z$. Therefore the six signed crosses define a hexagon in $Z$, and symmetry considerations force it to be regular.

We recall the notion of the divisor of an automorphic form. Let $Y \subset X$ be an irreducible subvariety of codimension one. We denote by $e_{Y}$ the ramification degree with respect to the natural projection $\pi: \mathcal{B}_{4} \rightarrow X$ (counted as 1 if $\pi$ is unramified along $Y$ ). If $Y$ is a short mirror this ramification degree is three, because the triflections are contained in $\Gamma$. For any other $Y$, such as a long mirror, it is one. If $F$ is a nonzero automorphic form for $\Gamma$ and the trivial character, then the vanishing order of $F$ along $\pi^{-1}(Y)$ is divisible by $e_{Y}$. We call the quotient of this vanishing order by $e_{Y}$ the order of $F$ along $Y$ and denote it by $n_{Y}(F)$. The divisor of $F$ in $X$ is the finite sum

$$
(F):=\sum_{Y \subset X} n_{Y}(F) Y
$$

We consider a cross in $X$ as a divisor with multiplicity one at all 5 of its mirrors. A fundamental result for this paper is

Theorem 4.6. Let $F \neq 0$ lie in the one dimensional space of automorphic forms associated to a cross. Then the divisor of $F$ in $X$ is exactly this cross. The 270 automorphic forms associated to the signed crosses have no common zeros in $\mathcal{B}_{4}^{*}$.

To prove this we will need a result whose proof we postpone to the next section. We remark that the form $\chi_{4}$ given here was first discovered by Borcherds [Bo3].

Theorem 4.7. There is an automorphic form $\chi_{4} \in[\operatorname{Aut}(\Lambda), 4, v]$ for some character $v$ of $\operatorname{Aut}(\Lambda)$, whose divisor in $\mathcal{B}_{4}$ is the sum of all the short mirrors, each with multiplicity one. Similarly, there exists $\chi_{75} \in\left[\operatorname{Aut}(\Lambda), 75, v^{\prime}\right]$ for some character $v^{\prime}$ of $\operatorname{Aut}(\Lambda)$, whose divisor in $\mathcal{B}_{4}$ is the sum of all the long mirrors, each with multiplicity one.

Proof of Theorem 4.6. Suppose the cross is $\left\{ \pm a_{0}, \ldots, \pm a_{4}\right\} \subset V$. If $\tilde{a}$ is a root of $\Lambda$ representing any of the $\pm a_{i}$, and $R$ is the biflection in $\tilde{a}$, then the relation $F \circ R=-F$ (which follows from the construction of $F$ ) implies that $F$ vanishes along the mirrors of $\tilde{a}$. Furthermore, if $\tilde{a}$ is a short root then $F$ is invariant 
under the triflection in $\tilde{a}$, so that the multiplicity in $\mathcal{B}_{4}$ is divisible by 3 . It follows that the divisor of $F$ in $\mathcal{B}_{4}$ contains the short mirrors of the cross with multiplicity 3 , plus the long mirrors of the cross. To prove the theorem it suffices to show that this is the full divisor of $F$. To see this we construct the product $P$ of all 270 automorphic forms, and divide $P$ by $\chi_{4}^{90} \chi_{75}^{6}$, where $\chi_{4}$ and $\chi_{74}$ are as in Theorem 4.7. The quotient is holomorphic because $P$ vanishes to order at least 6 along each long mirror in $\mathcal{B}_{4}$ and least $270 \cdot 3 \cdot 4 / 36=90$ along each short mirror. The quotient has weight $270 \cdot 3-90 \cdot 4-75 \cdot 6=0$, so is constant. It is nonzero because each $F$ is nonzero. Therefore the divisor of $P$ is the same as that of $\chi_{4}^{90} \chi_{75}^{6}$; since this is also the sum of the "known" divisors of the various $F$, the first statement of the theorem follows. The second follows immediately from this and Theorem 3.4 .

\section{Borcherds products and the proof of Theorem 4.7}

We recall some facts about automorphic forms on $\mathrm{O}(2, n)$, where $\mathrm{O}(2, n)$ is the orthogonal group of a real vector space $V$ with a symmetric bilinear form $(\cdot, \cdot)$ of signature $(2, n)$. Let $\mathcal{H}_{n}$ denote the hermitian symmetric space associated to $\mathrm{O}(2, n)$. It can be realized as an open subset of the quadric defined by $(z, z)=0$ in the projective space $P(V(\mathbb{C}))$, where we extend $(\cdot, \cdot)$ to a $\mathbb{C}$-bilinear form on $V(\mathbb{C})$. Namely, it is one of the two connected components of the open subset defined by $(z, \bar{z})>0$. A subgroup $\mathrm{O}^{\prime}(V)$ of index two of the orthogonal group $\mathrm{O}(V)$ acts biholomorphically on $\mathcal{H}_{n}$. Let $\tilde{\mathcal{H}}_{n}$ denote the inverse image of $\mathcal{H}_{n}$ in $V(\mathbb{C})$. We restrict henceforth to the case $n>2$ for convenience. If $M$ is an even integral $\mathbb{Z}$-lattice in $V$, then a meromorphic automorphic form of weight $k \in \mathbb{Z}$ with respect to a subgroup $G$ of finite index in

$$
\mathrm{O}^{\prime}(M)=\mathrm{O}(M) \cap \mathrm{O}^{\prime}(V)
$$

and a character $v$ of $G$ is a meromorphic function $f$ on $\tilde{\mathcal{H}}_{n}$ with the properties

$$
\begin{gathered}
f(\gamma z)=v(\gamma) f(z) \text { for all } \gamma \in G, \text { and } \\
f(t z)=t^{-k} f(z) \text { for all } t \in \mathbb{C}^{\bullet} .
\end{gathered}
$$

We next recall the notion of a Heegner divisor: let $m$ be a negative rational number and let $\alpha$ be an element of $M^{\prime} / M$, where $M^{\prime}$ denotes the dual lattice. The Heegner divisor $H(\alpha, m) \subset \mathcal{H}_{n}$ is the union of the orthogonal complements $v^{\perp} \cap \mathcal{H}_{n}$ where $v$ runs through all elements of $M^{\prime}$ satisfying

$$
v \equiv \alpha \bmod M \quad \text { and } \quad(v, v)=2 m .
$$

We consider $H(\alpha, m)$ as a divisor on $\mathcal{H}_{n}$ by attaching multiplicity 1 to all components. It is obvious that $H(\alpha, m)=H(-\alpha, m)$, so that the divisor depends only on $m$ and the image of $\alpha$ in $\left(M^{\prime} / M\right) / \pm 1$.

Borcherds introduced in [Bo1] a method for constructing automorphic forms on $\mathcal{H}_{n}$ whose divisors are sums of Heegner divisors. Then, in [Bo2], he constructed 
a 'space of obstructions' to the use of this technique for constructing automorphic forms with divisor equal to some given sum of Heegner divisors. This space consists of all elliptic modular forms of weight

$$
k:=(2+n) / 2
$$

with respect to the dual $\varrho^{*}:=\varrho_{M}^{*}$ of the Weil representation. We restrict to the case of even $n$ since the Weil representation simplifies and this is the only case we need. Such a form $\left(f_{\alpha}\right)_{\alpha \in M^{\prime} / M}$ is required to be holomorphic at the cusp at infinity and satisfy the transformation laws

$$
\begin{gathered}
f_{\alpha}(\tau+1)=e^{-\pi \mathrm{i}(\alpha, \alpha)} f_{\alpha}(\tau) \\
f_{\alpha}\left(-\frac{1}{\tau}\right)=-\sqrt{\frac{\tau}{\mathrm{i}}} \frac{1}{\sqrt{\left|M^{\prime} / M\right|}} \sum_{\beta \in M^{\prime} / M} e^{2 \pi \mathrm{i}(\alpha, \beta)} f_{\beta}(\tau) .
\end{gathered}
$$

As in section 4 we note that $(\alpha, \alpha)$ is well-defined modulo 2 and $(\alpha, \beta)$ is welldefined modulo 1, so that these formulas make sense.

Elements of the space of obstructions can be constructed by means of Eisenstein series, as follows. We write $R$ for the group ring $\mathbb{C}\left[M^{\prime} / M\right]$ and $R_{0}$ for the subspace on which $(-1)^{k} \varrho^{*}(-I)$ acts trivially. Since $(-1)^{k} \varrho^{*}(-I)$ acts by exchanging $e_{\alpha}$ and $e_{-\alpha}$, where the $e_{\alpha}$ form the standard basis of $R$ as $\alpha$ varies over $M^{\prime} / M$, a basis for $R_{0}$ is given by the elements

$$
e_{\alpha}+e_{-\alpha}, \quad \alpha \in\left(M^{\prime} / M\right) / \pm 1
$$

If $\xi \in R_{0}$ satisfies

$$
\varrho^{*}\left(\begin{array}{ll}
1 & 1 \\
0 & 1
\end{array}\right) \xi=\xi
$$

then $(c \tau+d)^{-k} \varrho^{*}(Q)^{-1} \xi$ remains unchanged if one replaces $Q$ by $P Q$, where $P= \pm\left(\begin{array}{ll}1 & n \\ 0 & 1\end{array}\right)$ lies in the stabilizer $\operatorname{SL}(2, \mathbb{Z})_{\infty}$ of $\infty$ and $Q=\left(\begin{array}{ll}a & b \\ c & d\end{array}\right) \in \operatorname{SL}(2, \mathbb{Z})$. This lets us define the Eisenstein series

$$
E_{\xi}(\tau)=\sum_{Q=\left(\begin{array}{ll}
a & b \\
c & d
\end{array}\right) \in \operatorname{SL}(2, \mathbb{Z})_{\infty} \backslash \operatorname{SL}(2, \mathbb{Z})}(c \tau+d)^{-k} \varrho^{*}(Q)^{-1} \xi,
$$

which is a modular form of weight $k$ with respect to $\varrho^{*}$, so it lies in the space of obstructions. Furthermore,

$$
\lim _{\operatorname{Im} \tau \rightarrow \infty} E_{\xi}(\tau)=\xi .
$$

In particular, if $\xi \neq 0$ then $E_{\xi}$ is not a cusp form.

Remark 5.1. Under our assumption $n>2$ we have $k>2$, and in this case the space of Eisenstein series of weight $k$ and with respect to $\varrho^{*}$ is isomorphic the space of all $\xi \in V_{0}$ with

$$
\varrho^{*}\left(\begin{array}{ll}
1 & 1 \\
0 & 1
\end{array}\right) \xi=\xi \quad\left(\text { and } \varrho^{*}(-I) \xi=(-1)^{k} \xi\right) \text {. }
$$


Using Remark 5.1 one can reformulate a fundamental result of Borcherds [Bo1], $[\mathrm{Bo} 2]$ as follows.

Theorem 5.2. Suppose $D$ is a finite $\mathbb{Z}$-linear combination

$$
\sum_{\alpha \in\left(M^{\prime} / M\right) / \pm 1, m<0} C(\alpha, m) H(\alpha, m)
$$

of Heegner divisors and $G$ is the subgroup of $\mathrm{O}^{\prime}(M)$ that acts trivially on $M^{\prime} / M$. Then $D$ is the divisor of a meromorphic automorphic form on $\mathcal{H}_{n}$ with respect to some character of $G$ if for every cusp form $f$ in the space of obstructions, say

$$
f_{\alpha}(\tau)=\sum_{m \in \mathbb{Q}} a_{\alpha}(m) \exp (2 \pi \mathrm{i} m \tau),
$$

the relation

$$
\sum_{m<0, \alpha \in M^{\prime} / M} a_{\alpha}(-m) C(\alpha, m)=0
$$

holds. The weight of such an automorphic form is

$$
\sum_{m \in \mathbb{Q}, \alpha \in M^{\prime} / M} b_{\alpha}(m) C(\alpha,-m),
$$

where $b_{\alpha}(m)$ denotes the Fourier coefficients of the (unique) Eisenstein series with constant term

$$
b_{\alpha}(0)= \begin{cases}-1 / 2 & \text { if } \alpha=0 \\ 0 & \text { otherwise }\end{cases}
$$

We want to apply this theorem to our lattice $\Lambda=\mathcal{E}^{1,4}$, or rather to its underlying $\mathbb{Z}$-lattice $M$. The obstructions have weight $k=(2+8) / 2=5$, and if the space of obstructions vanished then the existence of the forms of Theorem 4.7 would follow easily from Theorem 5.2 by restriction from $\mathcal{H}_{8}$ to $\mathcal{B}_{4}$. There are obstructions, and even cuspidal obstructions, but we will show that there are no $\mathrm{O}^{\prime}(M)$-invariant cusp forms in the space of obstructions, and this turns out to be enough to establish Theorem 4.7. Here, $\mathrm{O}^{\prime}(M)$ acts via its action on $M^{\prime} / M$.

Theorem 5.3. For $M$ equal to the $\mathbb{Z}$-lattice underlying $\Lambda=\mathcal{E}^{1,4}$, the space of $\mathrm{O}^{\prime}(M)$-invariant obstructions has dimension two and is spanned by Eisenstein series. The space of invariant cuspidal obstructions vanishes.

Proof. The $\mathrm{O}^{\prime}(M)$-invariant part of $\mathbb{C}\left[M^{\prime} / M\right]$ has dimension 4 , because $\mathrm{O}^{\prime}(M)$ acts with 4 orbits (or 'types') on $M^{\prime} / M$. The type of an element $\alpha \in M^{\prime} / M$ is defined as 00 if $\alpha$ is the zero element and as $t \in\{0,1,2\}$ if $\alpha$ is different from zero and $(\alpha, \alpha) \equiv 2 t / 3 \bmod 2$. There are 1, 80, 90 and 72 elements of $M^{\prime} / M$ of types 00, 0, 1 and 2, respectively. We will express an invariant obstruction $h$ as $\left(h_{00}, h_{0}, h_{1}, h_{2}\right)$, where each $h_{t}$ is the sum of the $h_{\alpha}$ as $\alpha$ varies over the elements 
of $M^{\prime} / M$ of type $t$. A calculation allows one to determine the action of $\operatorname{SL}(2, \mathbb{Z})$ with respect to this basis. It turns out that the standard generators $T=\left(\begin{array}{ll}1 & 1 \\ 0 & 1\end{array}\right)$ and $S=\left(\begin{array}{cc}0 & -1 \\ 1 & 0\end{array}\right)$ act by

$$
\varrho^{*}(T)=\left(\begin{array}{llll}
1 & & & \\
& 1 & & \\
& & \omega^{2} & \\
& & & \omega
\end{array}\right) \quad \text { and } \quad \varrho^{*}(S)=\frac{\mathrm{i}}{3^{5 / 2}}\left(\begin{array}{cccc}
1 & 1 & 1 & 1 \\
80 & -1 & 8 & -10 \\
90 & 9 & -9 & 0 \\
72 & -9 & 0 & 9
\end{array}\right) .
$$

Borcherds [Bo2] gives a formula for the dimension of the space of elliptic modular forms of given weight that transform according to some given representation of $\operatorname{SL}(2, \mathbb{Z})$, in terms of the eigenvalues of certain elements of $\mathrm{SL}(2, \mathbb{Z})$. Applying this formula to the 4-dimensional representation above shows that the space of obstructions has dimension 2. On the other hand, the space of Eisenstein series is also 2-dimensional, because another calculation shows that the subspace of $\mathbb{C}\left[M^{\prime} / M\right]^{\mathrm{O}^{\prime}(M)}$ whose elements satisfy the conditions of Remark 5.1 is 2-dimensional. Since a cuspidal Eisenstein series vanishes identically, the theorem follows.

Corollary 5.4. With $M$ as in Theorem 5.3, every divisor $D$ as in Theorem 5.2 that is $\mathrm{O}^{\prime}(M)$-invariant is the divisor of a form on $\mathcal{H}_{8}$ that is automorphic with respect to some character of $\mathrm{O}^{\prime}(M)$.

Proof. Since $D$ is $\mathrm{O}^{\prime}(M)$-invariant it satisfies condition (5.2) for all $f$ as in (5.1) if and only if it satisfies the condition for all such $f$ that are also $\mathrm{O}^{\prime}(M)$-invariant. Therefore the theorem assures us of the existence of an automorphic form for $G \subseteq \mathrm{O}^{\prime}(M)$ with divisor $D$, and since $D$ is $\mathrm{O}^{\prime}(M)$-invariant the form must be automorphic with respect to some character of $\mathrm{O}^{\prime}(M)$ itself.

In order to find the weights of the forms constructed in this way, we need the Eisenstein series with constant term $b_{\alpha}(0)=-1 / 2$ for $\alpha=0$ and $b_{\alpha}(0)=0$ for other $\alpha$. To compute this series we construct a basis for the space of Eisenstein series and then take a suitable linear combination. The Weil representation factors through $\mathrm{SL}(2, \mathbb{Z} / 3 \mathbb{Z})$, so our Eisenstein series are linear combinations of the classical elliptic Eisenstein series of level 3, namely

$$
G_{k}(\tau ; c, d, N):=\sum_{\substack{m \equiv c \\ n \equiv d}}^{\prime} \frac{1}{(m \tau+n)^{k}},
$$

where the level $N$ is 3 and the weight $k$ is 5 . We write $E_{1}, E_{2}, E_{3}$ and $E_{4}$ for the four classical Eisenstein series corresponding to the values $(c, d)=(0,1),(1,0)$, $(1,1)$ and $(1,2)$. We will continue to use the notation introduced in the proof of Theorem 5.3.

Proposition 5.5. With $M$ as in Theorem 5.3, a basis for the space of $\mathrm{O}^{\prime}(M)$ invariant obstructions consists of the Eisenstein series $f$ and $g$ given by 


$$
\begin{aligned}
f_{00} & =\frac{\mathrm{i} \sqrt{3}}{18} E_{1}-\frac{1}{18}\left(E_{2}+E_{3}+E_{4}\right) \\
f_{0} & =-\frac{5 \mathrm{i} \sqrt{3}}{9} E_{1}+\frac{5}{9}\left(E_{2}+E_{3}+E_{4}\right) \\
f_{1} & =0 \\
f_{2} & =E_{2}+\omega^{2} E_{3}+\omega E_{4} \\
g_{00} & =\frac{\mathrm{i} \sqrt{3}}{18} E_{1}+\frac{1}{18}\left(E_{2}+E_{3}+E_{4}\right) \\
g_{0} & =\frac{4 \mathrm{i} \sqrt{3}}{9} E_{1}+\frac{4}{9}\left(E_{2}+E_{3}+E_{4}\right) \\
g_{1} & =E_{2}+\omega E_{3}+\omega^{2} E_{4} \\
g_{2} & =0
\end{aligned}
$$

Proof. If $h=\left(h_{00}, h_{0}, h_{1}, h_{2}\right)$ is an $\mathrm{O}^{\prime}(M)$-invariant Eisenstein series then each component of $h$ is a $\mathbb{C}$-linear combination of $E_{1}, \ldots, E_{4}$. The manner in which the $E_{i}$ 's transform into each other under $\operatorname{SL}(2, \mathbb{Z})$ is known, and the transformation laws of $h$ with respect to $\varrho^{*}$ reduce to a set of linear conditions on the coefficients of the $E_{i}$ 's. One then solves the system of linear equations. (Of course, once one has the answer one can simply check it.)

The Fourier coefficients of the Eisenstein series can be found in many text books, for example [He, no. 24, section 1] or [Fr]. This lets one find the Fourier expansions for $f$ and $g$; once these are known then one can find the unique obstruction $h$ whose Fourier coefficients $b_{\alpha}(m)$ have constant term as in Theorem 5.2. The answer turns out to be given by

$$
\begin{aligned}
h_{00} & =-1 / 2+12 q+225 q^{2}+1092 q^{3}+2892 q^{4}+\ldots \\
h_{0} & =1080 q+16200 q^{2}+87480 q^{3}+260280 q^{4}+673920 q^{5}+\ldots \\
h_{1} & =225 q^{2 / 3}+9360 q^{5 / 3}+57825 q^{8 / 3}+219600 q^{11 / 3}+540450 q^{14 / 3}+\ldots \\
h_{2} & =12 q^{1 / 3}+2892 q^{4 / 3}+28824 q^{7 / 3}+112320 q^{10 / 3}+342744 q^{13 / 3}+\ldots .
\end{aligned}
$$

Proposition 5.6. With $M$ as in Theorem 5.3, there exists an automorphic form on $\mathcal{H}_{8}$ for $\mathrm{O}^{\prime}(M)$, of weight 12 (resp. 225), whose zeros are the orthogonal complements of the vectors $v \in M^{\prime}$ satisfying $(v, v)=-2 / 3 \quad(\operatorname{resp} .(v, v)=-1 / 3)$. The vanishing order is one.

Proof. This follows from Theorems 5.2 and 5.3. For the form of weight 12 (resp. 225) we take $D$ to be the sum of the $H(\alpha, m)$ where $m=-1 / 3$ (resp. $m=-2 / 3$ ) and $\alpha$ varies over the type 2 (resp. type 1 ) elements of $\left(M^{\prime} / M\right) / \pm 1$. 
We note that the form of weight 12 was found by Borcherds in [Bo3].

Proof of Theorem 4.7. We use the natural embedding $\mathrm{U}(1,4) \hookrightarrow \mathrm{O}^{\prime}(2,8)$ and a compatible holomorphic embedding $\mathcal{B}_{4} \hookrightarrow \mathcal{H}_{8}$. The short (resp. long) mirrors in $\mathcal{B}_{4}$ are the intersections of the divisors described in Prop. 5.6. The orthogonal complements in Prop. 5.6 occur in triples having the same intersection with $\mathcal{B}_{4}$, since if $r$ is a root of $\Lambda$ then $r, \omega r$ and $\omega^{2} r$ are roots with the same orthogonal complement in $\mathcal{B}_{4}$ but different orthogonal complements in $\mathcal{H}_{8}$. Therefore the vanishing order of the restriction to $\mathcal{B}_{4}$ along each short (resp. long) mirror is three. Taking a cube root yields a form of weight $12 / 3=4$ (resp. $225 / 3=45$ ).

\section{A model for the moduli space of marked cubic surfaces}

Recall the ten dimensional space $W$ of automorphic forms for $\Gamma$, the congruence subgroup of level $\sqrt{-3}$ in $\operatorname{Aut}(\Lambda)$. We know from Theorem 4.6 that these forms have no common zero. Therefore, by choosing a basis for $W$ we obtain an everywhere holomorphic map

$$
\beta: X=\mathcal{B}_{4}^{*} / \Gamma \longrightarrow P^{9}(\mathbb{C}) .
$$

This map is algebraic by Chow's theorem. By a result of Hilbert it is a finite map. Hence the image is a projective algebraic variety $\mathcal{V} \subset P^{9}$ of dimension 4 . In fact more is true:

Theorem 6.1. The map $\beta: X \rightarrow \mathcal{V}$ is birational.

After proving this theorem we will introduce a family of cubic 8-folds, each of which contains $\mathcal{V}$. Then we will sketch a proof that these cubic equations actually define $\mathcal{V}$. Our proof of Theorem 6.1 uses only our automorphic forms. In section 7 we will prove that $\beta$ is actually an embedding, but this relies heavily on the very extensive calculations and involved arguments of [Na]. Theorem 6.1 follows immediately from the lemma:

Lemma 6.2. Let $p$ be the point of $\mathcal{B}_{4}$ represented by $(1,0,0,0,0) \in \Lambda$, and let $\bar{p}$ denote its image in $X$. Then $\bar{p}$ is the only point of $X$ mapping to $\beta(\bar{p})$, and $\beta: X \rightarrow \mathcal{V}$ is a local diffeomorphism at $\bar{p}$.

Proof. The first claim is a consequence of the second part of Theorem 3.4. In order to prove the second claim we will find four elements of $W$, the sum of whose divisors in $X$ is a normal crossing divisor at $\bar{p}$. For this we will need coordinates around $\bar{p}$. Coordinates around $p \in \mathcal{B}_{4}$ may be taken to be $z_{1}, \ldots, z_{4} \in \mathbb{C}^{4}$, with $\sum_{i}\left|z_{i}\right|^{2}<1$ as in formula (3.1). The stabilizer $\Gamma_{p}$ of $p$, which is generated (modulo scalars) by the triflections in the short roots

$$
(0,1,0,0,0),(0,0,1,0,0),(0,0,0,1,0) \text { and }(0,0,0,0,1)
$$


acts by multiplying the $z_{i}$ by cube roots of unity. It follows that local coordinates for $X$ near $\bar{p}$ are given by the functions $w_{i}=z_{i}^{3}$. The four short mirrors of $X$ passing through $\bar{p}$ are given by the equations $w_{i}=0$. The long mirrors in $\mathcal{B}_{4}$ that pass through $p$ are the mirrors of the 216 roots $\left(0, a_{1}, \ldots, a_{4}\right)$, where two of the $a_{i}$ are zero and the others are sixth roots of unity. To work out their images in $X$ it suffices to treat the case where the nonzero $a_{i}$ lie in $\{ \pm 1\}$, since the orbit of these under $\Gamma_{p}$ is the entire set of 216. It is easy to check that the mirror $z_{i}= \pm z_{j}$ in $\mathcal{B}_{4}$ projects to the mirror $w_{i}= \pm w_{j}$ in $X$. It follows that in our local coordinates in $X$, the 12 long mirrors through $\bar{p}$ are given by the equations $w_{i}= \pm w_{j}$ for the various pairs $i \neq j$.

We claim that for each long mirror $m$ of $X$ passing through $\bar{p}$, there is a cross $C$ containing it whose short mirrors do not pass through $\bar{p}$. To see this, consider the three crosses containing $m$. Because only two of the short mirrors passing through $\bar{p}$ are orthogonal to $m$, one of the three crosses contains neither of these mirrors. Since it cannot contain either of the other short mirrors, it has the desired property and we take it to be $C$. Now, it is easy to find four long mirrors $m_{1}, \ldots, m_{4}$ whose sum is a normal crossing divisor at $\bar{p}$, for example those given by $w_{1}= \pm w_{2}$ and $w_{3}= \pm w_{4}$. We let $C_{i}$ be crosses associated to the $m_{i}$ as above, and $f_{i}$ be automorphic forms associated to the $C_{i}$. Then the $f_{i}$ are necessarily linearly independent, and we extend them to a basis $f_{1}, \ldots, f_{10}$ of $W$. Of course, one of the $f_{i}$, say $f_{10}$, does not vanish at $\bar{p}$, and then $f_{1} / f_{10}, \ldots, f_{9} / f_{10}$ are affine coordinates near $\beta(\bar{p}) \in P^{9}$. It follows from the implicit function theorem and the fact that $f_{i}(i=1, \ldots, 4)$ has only a simple zero along $m_{i}$ that $\beta$ is a local diffeomorphism as $\bar{p}$.

Next we will find some cubic relations satisfied by our automorphic forms; these define cubic 8-folds in $P^{9}(\mathbb{C})$ which contain $\mathcal{V}$. It is easy to explain the origin of these relations: it can happen that there are three crosses $C_{1}, C_{2}$ and $C_{3}$, and another three crosses $C_{1}^{\prime}, C_{2}^{\prime}$ and $C_{3}^{\prime}$, such that as divisors in $X$ they satisfy

$$
C_{1}+C_{2}+C_{3}=C_{1}^{\prime}+C_{2}^{\prime}+C_{3}^{\prime} .
$$

If $F_{i}$ and $F_{i}^{\prime}$ are nonzero automorphic forms in the one-dimensional subspaces of $W$ associated to the $C_{i}$ and $C_{i}^{\prime}$, then the divisors of $F_{1} F_{2} F_{3}$ and $F_{1}^{\prime} F_{2}^{\prime} F_{3}^{\prime}$ are equal and therefore the two products coincide up to a scalar. This relation would be trivial if the $C_{i}^{\prime}$ were obtained by permuting the $C_{i}$, but nontrivial relations do arise and can be found by studying the geometry of $V$. Here are some nontrivial cubic relations, which turn out to be the only ones. (Only trivial relations can be found if one plays the same game with pairs rather than triples of crosses.)

Lemma 6.3. Let $\left(a_{1}, a_{2}, a_{3}, b_{1}, b_{2}\right)$ be an ordered orthonormal basis of $V$, i.e., an orthogonal basis of norm 1 vectors. Let $S_{i}$ be the signed cross given by the basis $\left\{a_{i}, a_{i+1} \pm b_{1}, a_{i-1} \pm b_{2}\right\}$, and $S_{i}^{\prime}$ be the signed cross given by $\left\{a_{i}, a_{i+1} \pm b_{2}, a_{i-1} \pm b_{1}\right\}$, where the subscript of $a_{i \pm 1}$ should be read modulo 3 . Writing $F_{i}$ and $F_{i}^{\prime}$ for the automorphic forms associated to $S_{i}$ and $S_{i}^{\prime}$, we have $F_{1} F_{2} F_{3}=F_{1}^{\prime} F_{2}^{\prime} F_{3}^{\prime}$. 
Proof. We write $C_{i}$ and $C_{i}^{\prime}$ for the crosses underlying $S_{i}$ and $S_{i}^{\prime}$. It is easy to check that (6.1) holds, and it follows that $F_{1} F_{2} F_{3}$ is a constant multiple of $F_{1}^{\prime} F_{2}^{\prime} F_{3}^{\prime}$. To determine the constant, let $\alpha$ be the isotropic vector $a_{1}+a_{2}+a_{3} \in V$ and let $\tilde{\alpha}$ be any primitive isotropic element of $\Lambda$ representing $\alpha$. Using the product formula of Lemma 4.2 , it is easy to see that the element of $\mathbb{C}[V]^{\mathrm{SL}\left(2, \mathbb{F}_{3}\right)}$ associated to each $S_{i}$ and $S_{i}^{\prime}$ has component 1 at $\alpha$. By the relationship between the values of elements of $W$ at cusps of $\tilde{\mathcal{B}}_{4}^{*}$ and at the corresponding elements of $V$ (see the proof of Prop. 4.4), all the $F_{i}$ and $F_{i}^{\prime}$ take the same value at $\tilde{\alpha}$. Therefore $F_{1} F_{2} F_{3}(\tilde{\alpha})=F_{1}^{\prime} F_{2}^{\prime} F_{3}^{\prime}(\tilde{\alpha})$, and so $F_{1} F_{2} F_{3}=F_{1}^{\prime} F_{2}^{\prime} F_{3}^{\prime}$.

Remarks. We will discuss coincidences among these relations, and the fact that they account for all the relations arising from crosses $C_{i}, C_{i}^{\prime}$ satisfying (6.1). If $\left(\hat{a}_{1}, \hat{a}_{2}, \hat{a}_{3}, \hat{b}_{1}, \hat{b}_{2}\right)$ is another ordered orthonormal basis for $V$, then the relations given by the two bases are essentially the same if

$$
\left\{ \pm a_{1}, \pm a_{2}, \pm a_{3}\right\}=\left\{ \pm \hat{a}_{1}, \pm \hat{a}_{2}, \pm \hat{a}_{3}\right\} \quad \text { and } \quad\left\{ \pm b_{1}, \pm b_{2}\right\}=\left\{ \pm \hat{b}_{1}, \pm \hat{b}_{2}\right\} .
$$

By "essentially the same" we mean that each relation implies the other. There are $|\mathrm{O}(5,3)| / 2^{5} 3 ! 2 !=270$ equivalence classes of ordered orthonormal bases under the relation (6.2), yielding 270 cubic relations. It is easy to make a computer enumerate all nontrivial pairs of triples of crosses $C_{i}$ and $C_{i}^{\prime}$ satisfying (6.1) and check that every one is a case of our construction. Therefore we have found all the relations arising from equalities of sums of triples of crosses. For convenience in enumerating the 270 relations, we remark that they are in 1-1 correspondence with the unordered triples of mutually orthogonal long mirrors in $X$. To find the relation associated to such a triple of mirrors, let $a_{1}, a_{2}$ and $a_{3}$ be long vectors of $V$ associated to the mirrors, extend them to an orthonormal basis of $V$, and apply the lemma.

Theorem 6.4. The variety $\mathcal{V}$ is the intersection of the cubic eightfolds defined by the relations of Lemma 6.3.

Proof sketch. Using one of the computer algebra systems MACAULAY or SINGULAR, it is easy to see that the dimension of the intersection $\mathcal{V}^{\prime}$ of the 270 cubics has dimension 4 . With either system it is possible to compute a projective resolution of $R / J$, where $R=\mathbb{Q}\left[Y_{0}, \ldots, Y_{9}\right], Y_{0}, \ldots, Y_{9}$ are a basis for $W$, and $J$ the ideal generated by the 270 cubic relations. The projective dimension of $R / J$ turns out to be 5 , by a calculation that takes a few minutes in SINGULAR but several hours in MACAULAY. As a consequence, $\mathcal{V}^{\prime}$ contains no component of dimension $<4$.

It is more involved to prove that $\mathcal{V}^{\prime}$ is irreducible. In principle one can simply ask the machine, but this seems to be too much for the computer. Instead, we consider the intersection of $\mathcal{V}^{\prime}$ with a hyperplane corresponding to a cross. If $\mathcal{V}^{\prime}$ is irreducible then the intersection should consist of 5 irreducible components. It is not hard to prove that in our situation the converse is also true. The hyper- 
plane section is defined by a certain ideal $\mathfrak{a} \subset \mathbb{C}\left[Y_{0}, \ldots, Y_{9}\right]$. In principle one can ask the computer for the components of the ideal (e.g. by using "decompose" in MACAULAY), but again this does not work. Instead, one finds directly five ideals $\mathfrak{a}_{0}, \ldots, \mathfrak{a}_{4}$ containing $\mathfrak{a}$ that come from the five mirrors of the cross and are constructed in an obvious way. After the ideals $\mathfrak{a}_{i}$ have been constructed, one can verify $\mathfrak{a}=\mathfrak{a}_{0} \cap \cdots \cap \mathfrak{a}_{4}$ by means of MACAULAY or SINGULAR. The problem now is to prove that the varieties of the $\mathfrak{a}_{i}$ are irreducible. This means that we face a similar problem in a lower dimension, which can be treated in a similar manner. During this procedure several very interesting ball quotients of smaller dimension occur. This will be treated in a separate paper, where more details about the ideal $J \subset \mathbb{C}\left[Y_{0}, \ldots, Y_{9}\right]$ and the hyperplane sections will be given.

We also intend to include proofs of the facts that $J$ is prime and that $R / J$ is normal. This has the important consequence that $W$ generates the ring of all automorphic forms on $\Gamma$ with trivial multipliers. The normality can be used to give an alternate proof of Corollary 7.3 (that $\beta$ is an embedding). We will also give the Hilbert function of $R / J$.

\section{Cross-ratios}

In this section we will relate our automorphic forms to the original invariants of a cubic surface, the cross-ratios of Cayley. These are rational functions on the moduli space of marked cubic surfaces that encode the manner in which the 27 lines on a cubic surface lie in $P^{3}$. We will show below that Cayley's cross-ratios are ratios of certain pairs of our 270 automorphic forms. Then we will use this to prove that the map $\beta: \mathcal{M} \rightarrow P^{9}$ of section 6 is an embedding.

Suppose that $A$ and $B$ are two crosses with the same long mirror $m$. By the proof of Lemma 4.5, the subspace of $W$ that changes sign under reflection in $m$ is 2-dimensional, and the automorphic forms coming from the six signed crosses of $m$ form a regular hexagon in this plane, centered at 0 . Now, $A$ and $B$ define two diameters of this hexagon, and we choose an endpoint $F$ (resp. $G$ ) of the diameter associated to $A$ (resp. $B$ ), such that $F$ and $G$ are adjacent vertices of the hexagon. There is a unique way to do this, up to simultaneously negating $F$ and $G$, so the rational function $F / G$ does not depend on the choice made. We call this the crossratio $A / B$. The reason for the name is Theorem 7.2 below, which identifies these rational functions with Cayley's cross-ratios. It is a happy and genuine accident of terminology that Cayley's cross-ratios may be regarded as ratios of crosses. There are 270 cross-ratios, six for each of the 45 long mirrors. To identify our cross-ratios with Cayley's we will need to describe the divisor of $A / B$ :

Lemma 7.1. If $m$ is a long mirror in $X$ and $A, B$ and $C$ are its three crosses, then the divisor of the cross-ratio $A / B$ consists of the four short mirrors 
of $A$ with multiplicity 1 (simple zeros) and the four short mirrors of $B$ with multiplicity -1 (simple poles). Furthermore, $A / B$ takes the constant value 1 along the short mirrors of $C$.

Proof. If $F$ and $G$ are automorphic forms chosen as in the discussion above, then their divisors in $X$ are the crosses $A$ and $B$, respectively. Since the long mirrors of $A$ and $B$ coincide and the short mirrors are distinct, the identification of the divisor of $A / B$ is complete. Finally, $H=F-G$ is an endpoint of the third diameter of the hexagon, so that it lies in the 1-dimensional subspace of $W$ associated to $C$, and in particular it vanishes on the short mirrors of $C$. That is, $F=G$ on the mirrors of $C$ and so $A / B=1$ along the short mirrors of $C$.

Now we discuss Cayley's cross-ratios; our basic reference is Naruki's extensive study of them and a compactification $C$ of the moduli space $M$ of marked smooth cubic surfaces that they define [Na]. The biregular action of $W\left(E_{6}\right)$ on $M$ extends to a biregular action on $C$, and the complement of $M$ in $C$ has 76 components, which fall into orbits of size 40 and 36 under $W\left(E_{6}\right)$. The components in the orbit of size 40 are all disjoint and can be blown down to points. The variety $\check{C}$ obtained by this blowing-down is the standard Geometric Invariant Theory (GIT) compactification of $M$, with its natural $W\left(E_{6}\right)$-action. Now, $M$ is also $W\left(E_{6}\right)$-equivariantly isomorphic to the complement in $X$ of the short mirrors, and the inclusion of this space into $X$ is also the standard GIT compactification. It follows that $X$ is $W\left(E_{6}\right)$-equivariantly isomorphic to $\check{C}$, with the 36 short mirrors corresponding to the images in $\breve{C}$ of the remaining 36 components of $C-M$.

Naruki describes $M$ in terms of a maximal torus $T$ of the simple Lie group $D_{4}$ of adjoint type. He writes $\Delta$ for the union of the subtori which are the fixedpoint sets of the 12 reflections of $W\left(D_{4}\right)$, and realizes $M$ as the blowup of $T$ at the identity element, minus the proper transforms of the 12 components of $\Delta$. He introduces multiplicative characters $\lambda, \mu, \nu$ and $\rho$ of $T$, which provide a coordinate system for $T$, and describes the action of $W\left(E_{6}\right)$ on $M$ by giving explicit rational self-maps of $T$ in terms of these coordinates. This group $W\left(E_{6}\right)$ contains the obvious group $W\left(D_{4}\right)$.

Naruki introduces 45 divisors in $M$ which $W\left(E_{6}\right)$ permutes transitively. One of these, $\delta_{0}$, is the exceptional divisor lying over the identity of $T$, and the rest are given by explicit equations in $\lambda, \mu, \nu$ and $\rho$. We claim that these 45 divisors correspond to our long mirrors. This follows because each of [Na] and [ACT2] proves that its set of 45 divisors represent the marked cubic surfaces that have an Eckardt point.

The passage from $T$ to $\check{C}$ involves compactifying $T$ and then performing a sequence of blowings-up and blowings-down. All that matters to us is that the identity of $T$ is blown up, and that the 12 components of $\Delta$ (or rather their transforms in $\breve{C}$ ) are among the the 36 components of $\breve{C}-M$. Naruki calls these 12 divisors the $A_{1}$-hypersurfaces. 
Finally, Naruki's table 2 gives 45 of Cayley's cross-ratios explicitly as rational functions of $\lambda, \mu, \nu$ and $\rho$. The full set of Cayley's 270 cross-ratios is obtained by following these functions by the 6 projective linear transformations of $P^{1}=\mathbb{C} \cup$ $\{\infty\}$ that preserve $\{0,1, \infty\}$. Of course, Cayley had much more explicit geometric concepts in mind when defining his cross-ratios; for details see Naruki's paper.

Theorem 7.2. Cayley's cross-ratios coincide with ours.

Proof. The idea is to check that the divisors coincide and that Cayley's crossratios satisfy the normalization condition of lemma 7.1. By Cayley's geometric considerations ([Na], §3), his cross-ratios do not take any of the values 0,1 and $\infty$ in $M$, so their divisors consist of short mirrors with some multiplicities. For the short mirror $S$ given by $\rho=1$ in Naruki's coordinates, simple substitution reveals the behavior along $S$ of the 45 cross-ratios given in Naruki's table. Namely, exactly 7 vanish along it, exactly 7 have poles along it, and just one takes the constant value 1 . Since the full set of Cayley's cross-ratios is obtained by following these by the 6 linear fractional transformations preserving $\{0,1, \infty\}$, we see that exactly $2 \cdot(7+7+1)=30$ of Cayley's cross-ratios vanish along $S$, another 30 take the constant value 1 , and a further 30 have poles along $S$. (Working with the full set of 270 restores the symmetry between 0,1 and $\infty$ that Naruki's choice of 45 conceals.) Now, by the transitivity of $W\left(E_{6}\right)$ on Cayley's cross-ratios, each vanishes along the same number, say $k$, of short mirrors. By transitivity on the short mirrors, each short mirror lies in the zero-locus of exactly 30 of Cayley's cross-ratios. These transitivities also show that $270 \cdot k=36 \cdot 30$, so that $k=4$ and each of Cayley's cross-ratios vanishes along exactly 4 short mirrors. The same argument also shows that each has poles along exactly 4 short mirrors.

Now we consider Cayley's cross-ratio $r(\mathrm{w})$, given in Naruki's coordinates by

$$
r(\mathrm{w})=\frac{(\lambda \rho-1)(\mu \rho-1)(\nu \rho-1)(\lambda \mu \nu \rho-1)}{(\mu \nu \rho-1)(\lambda \nu \rho-1)(\lambda \mu \rho-1)(\rho-1)} .
$$

We will write simply $r$ for $r(\mathrm{w})$. The sets $\chi=1$, where $\chi$ is one of the characters $\lambda \rho, \mu \rho, \nu \rho$ and $\lambda \mu \nu \rho$ (resp. $\mu \nu \rho, \lambda \nu \rho, \lambda \mu \rho$ and $\rho$ ) appearing in the numerator (resp. denominator) are among Naruki's $A_{1}$-hypersurfaces, so $r$ has a simple zero (resp. simple pole) along these four short mirrors. By the argument above, these constitute the entire divisor of $r$. Furthermore, the short mirrors along which $r$ vanishes (resp. has a pole) are orthogonal, in the sense that the reflections across them commute. To see this we do not even need to perform a calculation, because Naruki (p. 20) has already organized his twelve $A_{1}$-hypersurfaces into three sets each consisting of four mutually orthogonal divisors. Finally, all 8 of these short mirrors are orthogonal to the long mirror $\delta_{0}$, because their reflections obviously commute with the negation map of $T$. It follows that $\delta_{0}$ together with the four short mirrors coming from the numerator (resp. denominator) of $r$ form a cross $C_{n}$ (resp. $C_{d}$ ). Therefore $r$ has the same divisor as our cross-ratio $C_{n} / C_{d}$. To show that $r=C_{n} / C_{d}$ it now suffices to show that $r=1$ along the short mirrors of the 
third cross associated to $\delta_{0}$. Consulting again the table on Naruki's p. 20, we see that these mirrors are given by $\chi=1$, where $\chi$ varies over the characters $\lambda, \mu, \nu$ and $\lambda \mu \nu \rho^{2}$. Simple calculation verifies the condition, so $r=C_{n} / C_{d}$. Since one of Cayley's cross-ratios coincides with one of ours, and $W\left(E_{6}\right)$ acts transitively on both sets of cross-ratios, the theorem follows.

Remark. B. van Geemen $[\mathrm{vG}]$ has also obtained this theorem, as a byproduct of a larger investigation. His idea is to construct and study the linear system on Naruki's model of the moduli space that comes from our space $W$ of automorphic forms. After one understands this linear system (van Geemen identifies it with one introduced by Coble [C] long ago), the result above follows immediately. His approach also has the advantage of allowing one to relate the moduli space $\mathcal{M}$ to the variety $\mathcal{V}$ over fields other than $\mathbb{C}$. (Note that $\mathcal{V}$ is defined over $\mathbb{Z}$.)

Corollary 7.3. The map $\beta: X \rightarrow P^{9}$ of section 6 is an embedding.

Proof. We write $\partial X$ for $\overline{\mathcal{B}_{4} / \Gamma}-\mathcal{B}_{4} / \Gamma$, the set of 40 cusp points. One of Naruki's main results is that the 270 cross-ratios, a priori defined as maps $M \rightarrow\left(P^{1}-\right.$ $\{0,1, \infty\})$, extend to regular maps $(X-\partial X) \rightarrow P^{1}$ that embed $X-\partial X$ in $\left(P^{1}\right)^{270}$. Since the cross-ratios are quotients of the elements of $W, \beta$ must embed $X-\partial X$ in $P^{9}$. Unfortunately, this argument cannot be extended to show that $\beta$ embeds all of $X$ into $P^{9}$; the problem is that one must blow up the points of $\partial X$ in order for the rational map from $X$ to $\left(P^{1}\right)^{270}$ to become regular. In order to prove the theorem we will first show that $\beta$ is injective as a map of sets, and then that $\beta$ is a local embedding at each point of $\partial X$.

The injectivity has essentially already been proven: Theorem 3.4 shows that for each $x \in \partial X, x$ is the only point of $X$ that lies on all the crosses containing $x$. It follows that no point of $\partial X$ is identified under $\beta$ with any other point of $X$. Since $\beta$ is already known to be injective on $X-\partial X, \beta$ is injective.

Now we prove that $\beta$ is a local embedding at each point of $\partial X$; we will use Naruki's explicit description (see [Na], section 12) of these singularities. Namely, his $T$-equivariant compactification $\tilde{T}$ of $T$ adjoins 48 divisors, 24 of which he then blows down to obtain 24 of the points of $\partial X$. Focusing on one of these divisors, which he denotes by $\overline{\rho=0}$ and we will denote by $D$, he gives 8 characters of $T$ which extend to regular functions $z_{1}, \ldots, z_{8}$ on a neighborhood $\mathcal{U}$ of $D$ in $\tilde{T}$, and which vanish along $D$. According to his theorem 12.1, the induced map $\mathcal{U} \rightarrow \mathbb{C}^{8}$ gives the blowing-down of $D$ and thus embeds a neighborhood of the resulting singular point $x \in \partial X$ into $\mathbb{C}^{8}$. Furthermore, he explicitly describes the singularity as the cone on the Veronese embedding of $P^{1} \times P^{1} \times P^{1}$ in $P^{7}$. This makes it a simple matter to see that the divisor of each $z_{i}$ near $x$ has exactly three components, and these components meet each other away from $x$ as well as at $x$. Since each $z_{i}$ is the extension of a character of $T$, its divisor can consist only of the components of $X-M$, which is to say, short mirrors. Since short mirrors that meet each other 
in $X-\partial X$ must be orthogonal, we have shown that the divisor of each $z_{i}$ near $X$ consists of three mutually orthogonal short mirrors. For each $i=1, \ldots, 8$, we will find an automorphic form $\psi_{i} \in W$ whose divisor near $x$ coincides with that of $z_{i}$. We may also choose $\psi^{\prime} \in W$ whose divisor misses $x$ entirely. Then the evaluation of $\psi_{1} / \psi^{\prime}, \ldots, \psi_{8} / \psi^{\prime}$ provides essentially the same map (of some neighborhood of $x)$ into $\mathbb{C}^{8}$ as Naruki's. It follows that $\beta$ must embed a neighborhood of $x$ into $P^{9}$.

All that remains is to show that if $x \in \partial X$ and $m_{1}, m_{2}$ and $m_{3}$ are any three mutually orthogonal short mirrors that all meet $x$, then there exists $\psi \in W$ whose divisor near $X$ is just the sum of the $m_{i}$. We choose a primitive null vector $v \in \Lambda$ representing $x$, and short roots $r_{i} \in v^{\perp}$ whose mirrors represent the $m_{i}$. Denoting the images of these vectors in $V$ by $\bar{x}$ and $\bar{r}_{i}$, we may choose coordinates in $V$ so that the inner product is given by

$$
(a, b)=a_{0} b_{0}-a_{1} b_{1}-\cdots-a_{4} b_{4},
$$

and $\bar{r}_{1}=(0,0,1,0,0), \bar{r}_{2}=(0,0,0,1,0), \bar{r}_{3}=(0,0,0,0,1)$ and $\bar{v}=(1,1,0,0,0)$. The standard cross, given by the pairs $( \pm 1,0,0,0,0), \ldots,(0,0,0,0, \pm 1)$, is the divisor of one of our Borcherds products, which we take to be $\psi$. It is obvious that the divisor of $\psi$ contains the $m_{i}$. To show that the other components of the divisor miss $x$, we observe that these components correspond to the orthogonal complements of the roots of $\Lambda$ whose images in $V$ are $( \pm 1,0,0,0,0)$ or $(0, \pm 1,0,0,0)$. Any such root has inner product $\not \equiv 0(\bmod 3)$ with $v$, so its mirror cannot contain $v$.

\section{References}

[ACT1] D. Allcock, J. Carlson and D. Toledo, A complex hyperbolic structure for the moduli of cubic surfaces, C.R.A.S. ser. I 326 (1988), 49-54.

[ACT2] D. Allcock, J. Carlson and D. Toledo, The complex hyperbolic geometry of the moduli space of cubic surfaces. To appear in J. Alg. Geom.; available at http:// www.math.harvard.edu/ allcock

[BB] W. L. Baily and A. Borel, Compactification of arithmetic quotients of bounded symmetric domains, Ann. Math. 84 (1966), 442-528.

[Bo1] R. Borcherds, Automorphic forms with singularities on Grassmannians, Invent. Math. 132 (1998), 491-562; available at http://www. math.berkeley.edu/ ${ }^{\sim}$ reb

[Bo2] R. Borcherds, The Gross-Kohnen-Zagier theorem in higher dimensions, Duke Math. J. 97 (1999), 219-233; available at http://www. math.berkeley.edu/ ${ }^{\sim}$ reb

[Bo3] R. Borcherds, An automorphic form related to cubic surfaces. Unpublished (1997); available at http://www. math.berkeley.edu/ reb

[C] A. B. Coble, Point sets and allied Cremona groups III, Trans. AMS 18 (1917), 331-372.

[Co] J. H. Conway et al., Atlas of Finite Groups, Oxford University Press, 1985.

[EHV] D. Eisenbud, C. Huneke and W. Vasconcelos, Direct methods for primary decomposition, Inv. Math. 110 (1992), 207-235.

[Fr] E. Freitag, Modular forms related to cubic surfaces, Available at http://www.rzuser.uniheidelberg. de ${ }^{\sim}$ t91

[He] E. Hecke, Mathematische Werke, Göttingen Vandenhoeck und Ruprecht, 1959.

[Na] I. Naruki, Cross ratio variety as moduli space of cubic surfaces, Proc. London Math. Soc. (3) 45 (1982), 1-30. 
[Sh] G. Shimura, The arithmetic of automorphic forms with respect to a unitary group, Ann. Math. 107 (1978), 596-605.

[vG] B. van Geemen, A linear system on Naruki's moduli space of marked cubic surfaces, to appear in Int. J. Math.

Daniel Allcock

Harvard University

Department of Mathematics

Cambridge, MA 02138

USA

e-mail: allcock@math.harvard.edu

URL: http://www.math.harvard.edu/ allcock
Eberhard Freitag

Mathematisches Institut

Im Neuenheimer Feld 288

D-69120 Heidelberg

Germany

e-mail: freitag@mathi.uni-heidelberg.de

URL: http://www.rzuser.uni-heidelberg.de/ ${ }^{\sim}$ t91

(Received: December 5, 2000)

(1) To access this journal online:

(40) http://www.birkhauser.ch 